\title{
Planar Josephson tunnel junctions in a transverse magnetic field
}

Monacoa, R.; Aarøe, Morten; Mygind, Jesper; Koshelets, V.P.

Published in:

Journal of Applied Physics

Link to article, DOI:

$10.1063 / 1.2809310$

Publication date:

2007

Document Version

Publisher's PDF, also known as Version of record

Link back to DTU Orbit

Citation (APA):

Monacoa, R., Aarøe, M., Mygind, J., \& Koshelets, V. P. (2007). Planar Josephson tunnel junctions in a transverse magnetic field. Journal of Applied Physics, 102(9), 093911. https://doi.org/10.1063/1.2809310

\section{General rights}

Copyright and moral rights for the publications made accessible in the public portal are retained by the authors and/or other copyright owners and it is a condition of accessing publications that users recognise and abide by the legal requirements associated with these rights.

- Users may download and print one copy of any publication from the public portal for the purpose of private study or research.

- You may not further distribute the material or use it for any profit-making activity or commercial gain

- You may freely distribute the URL identifying the publication in the public portal

If you believe that this document breaches copyright please contact us providing details, and we will remove access to the work immediately and investigate your claim. 


\title{
Planar Josephson tunnel junctions in a transverse magnetic field
}

\author{
R. Monaco ${ }^{\text {a) }}$ \\ Istituto di Cibernetica del C.N.R., 80078, Pozzuoli, Italy \\ and Unita' INFM-Dipartimento di Fisica, Universita' di Salerno, 84081 Baronissi, Italy
}

M. Aaroe ${ }^{\text {b) }}$ and J. Mygind

Department of Physics, B309, Technical University of Denmark, DK-2800 Lyngby, Denmark

V. P. Koshelets ${ }^{\text {) }}$

Institute of Radio Engineering and Electronics, Russian Academy of Science, Mokhovaya 11, Bldg 7, 125009, Moscow, Russia

(Received 27 August 2007; accepted 26 September 2007; published online 13 November 2007)

\begin{abstract}
Traditionally, since the discovery of the Josephson effect in 1962, the magnetic diffraction pattern of planar Josephson tunnel junctions has been recorded with the field applied in the plane of the junction. Here we discuss the static junction properties in a transverse magnetic field where demagnetization effects imposed by the tunnel barrier and electrodes geometry are important. Measurements of the junction critical current versus magnetic field in planar Nb-based high-quality junctions with different geometry, size, and critical current density show that it is advantageous to use a transverse magnetic field rather than an in-plane field. The conditions under which this occurs are discussed. (c) 2007 American Institute of Physics. [DOI: 10.1063/1.2809310]
\end{abstract}

\section{INTRODUCTION}

It is well known that a magnetic field $\mathbf{H}$ modulates the critical current $I_{c}$ of a Josephson tunnel junction (JTJ). ${ }^{1}$ Indeed the occurrence of such diffraction phenomena $I_{c}(H)$ is one of the most striking behaviors of JTJs. ${ }^{2}$ In the case of a planar JTJ, it was Josephson himself ${ }^{3}$ who pointed out that the gradient of the Josephson phase $\phi$, which is the difference between the complex wave function phases in the electrodes, can be expressed as

$$
\boldsymbol{\nabla} \phi=\frac{2 \pi d_{e} \mu_{0}}{\Phi_{0}} \mathbf{H} \times \mathbf{n}
$$

where $\mathbf{n}$ is a unit vector normal to the insulating barrier separating the two superconducting electrodes, $\mu_{0}$ is the vacuum permeability, and $\Phi_{0}=h / 2 e$ is the magnetic flux quantum. If the two superconducting films have thicknesses $d_{1,2}$, London penetration depths $\lambda_{L 1,2}$, and $t_{j}$ is the barrier thickness, then the effective magnetic penetration $d_{e}$ is given by $^{4}$

$$
d_{e}=t_{j}+\lambda_{L 1} \tanh \frac{d_{1}}{2 \lambda_{L 1}}+\lambda_{L 2} \tanh \frac{d_{2}}{2 \lambda_{L 2}},
$$

which, in the case of thick superconducting films $\left(d_{i} \gg \lambda_{L i}\right)$, reduces to $d_{e} \approx \lambda_{L 1}+\lambda_{L 2}$.

Since Rowell ${ }^{5}$ in 1963 made the first experimental verification of Eq. (1), a large number of theoretical and experimental papers have been devoted to the study of magnetic diffraction patterns, in various Josephson junctions. Nowadays the $I_{c}(H)$ curves for planar JTJs having the most common geometrical and electrical parameters are fully understood (see, for example, Chaps.4 and 5 of Ref. 1). It is

\footnotetext{
a) Electronic mail: roberto@sa.infn.it

${ }^{b)}$ Electronic mail: aaroe@fysik.dtu.dk

${ }^{c)}$ Electronic mail: valery@ hitech.cplire.ru
}

important to point out that nearly all work was done with the external magnetic field applied in the barrier plane. In fact, since Eq. (1) states that $\phi$ is insensitive to transverse fields, this is the most obvious choice of the magnetic field orientation.

For the earlier reasons, a magnetic field parallel to the barrier of planar JTJs is applied in the practical applications of the Josephson magnetic diffraction phenomena such as, for example, the suppression of the direct current Josephson effect in superconductor-insulator-superconductor mixers for photon detection ${ }^{6}$ and in specially shaped JTJs for particle detection, ${ }^{7}$ the magnetic biasing of a flux flow oscillator, ${ }^{8}$ and the tuning of resonant fluxon oscillators. ${ }^{9}$

\section{TRANSVERSE MAGNETIC FIELD}

In 1975 Rosenstein and Chen ${ }^{10}$ first reported on the effect of a transverse magnetic field on the critical current of a JTJ with overlap geometry. Among other things, they showed that the value of the junction critical field $H_{c}$ at which the magnetic diffraction pattern first goes to zero, changes with the inclination of the field with respect to the barrier plane, the minimum being obtained when the field is transverse. This was the first experimental observation that transverse fields could be more efficient that parallel ones in modulating the Josephson current. Soon after, Hebard and Fulton ${ }^{11}$ correctly interpreted the findings of Ref. 10 in terms of stationary screening currents which develop when a superconductor is subjected to an external magnetic field. To better understand the mechanism through which also a transverse field is able to modulate the critical current of a planar JTJ, let us consider first a single isolated superconducting film immersed in a uniform static magnetic field $H_{\perp}$ perpendicular to its surface. This system has received a continuous interest over the years and here we only recall the main features. For a deep treatment of this topic we remand to Ref. 12, and 
references therein. We assume that the film thickness $d$ is larger than its London penetration depth $\lambda_{L}$ and that the field everywhere is much smaller than the critical field which would force the film into the intermediate or normal state, i.e., that the film is in the flux-free Meissner regime. At the top and bottom film surfaces, the flux lines are excluded from the interior of the film where $\mathbf{H}=0$. In fact, due to the screening currents $\mathbf{J}_{\mathbf{s}}(=\boldsymbol{\nabla} \times \mathbf{H})$, they bend as they approach the film surface, flow along the film surfaces, concentrate at the film edges, and bent backward. Due to continuity, $H_{n}$, the component of $\mathbf{H}$ normal to the surface, may be taken to be zero, while its tangential component $H_{t}$ decays exponentially inside the film on the scale of $\lambda_{L}$.

The knowledge of the distribution of the magnetic field lines around the film requires a self-consistent solution of a magnetostatic problem combining the London equation $(\mathbf{H}$ $\left.+\lambda_{L}^{2} \nabla^{2} \mathbf{H}=0\right)$ in the superconducting film and the fourth Maxwell equation $(\boldsymbol{\nabla} \times \mathbf{H}=0)$ in the empty space around the film with boundary conditions appropriate to the film surface geometry. This problem can be solved analytically only for simple axially symmetric cases such as, for example, that of an ellipsoid of revolution with the axis of revolution parallel to the applied field $H_{\perp} \cdot{ }^{13}$

If the film width $w$ is much less than its length, but much greater than its thickness $d$, then we can approximate the film as a elliptical oblate cylinder of infinite length whose cross section has axes $w$ and $d$; with the applied magnetic field $H_{\perp}$ directed along the minor axis, then $H_{t}$, the component of $\mathbf{H}$ tangent to the surface, only depends on the angle $\beta$ with respect to the minor axis: $H_{t} / H_{\perp}=1 / \sqrt{ } \cos ^{2} \beta+(d / w)^{2} \sin ^{2} \beta$, whose maximum value $w / d \gg 1$ occurs at the cylinder edges $(\beta= \pm \pi / 2) .{ }^{14}$ If the film width $w$ is comparable to its length, the film can be approximated by a disk whose diameter $w$ is much greater than its thickness $d$, with the external field applied parallel to its axis. In this case, for symmetry reasons, on the disk top and bottom surfaces the magnetic field lines are radial and $H_{t}$ only depends on the distance $r$ from the disk center; it is null at the center of the disk and increases as we move outward. ${ }^{15}$ The surface or sheet current density $\mathbf{j}_{\mathbf{s}}$, defined as the screening current density $\mathbf{J}_{\mathbf{s}}$ integrated over the specimen thickness, equals in magnitude $H_{t}$ and is everywhere orthogonal to $\mathbf{H}$. Numerical simulations carried out for a $\mathrm{Nb}$ disk having $\lambda_{L}=90 \mathrm{~nm}$ show that the shape of $H_{t}(r)$ only depends on the disk aspect ratio $w / d$, as far as $d \gg \lambda_{L}$. We found that at the disk border $H_{t}$ is several times larger than the applied field $H_{\perp}$, as shown in Fig. 1 for three values of the disk aspect ratio $w / d=10,100$, and 1000, with $d=1 \mu \mathrm{m}$. Both film approximations lead one to conclude that a thin superconducting film of any geometry in a transverse field produce a magnetic field: (i) whose orientation on the film end surfaces is parallel to surfaces themselves; (ii) whose direction near the borders of the film end surfaces is perpendicular to the borders themselves; and (iii) whose strength is proportional to the transverse applied field intensity and exceeds its value near the borders of a film with a large aspect ratio $w / d$.

Now, we can consider the situation in which two superconducting films partially overlap to form a planar JTJ. If the tunnel barrier is very transparent, then the screening currents

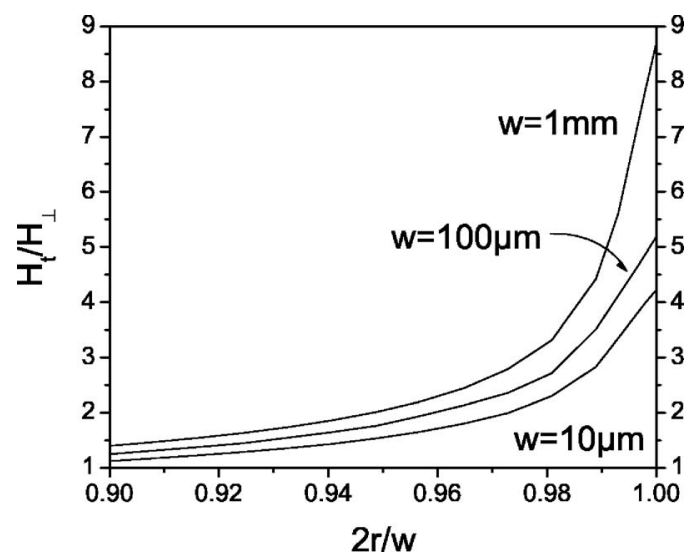

FIG. 1. Computed normalized magnetic induction field $H_{t} / H_{\perp}$ at the border of a $\mathrm{Nb}$ superconducting disk in a perpendicular field $H_{\perp}$. The disk thickness is $d=1 \mu \mathrm{m}$ and diameters $w=10,100$, and $1000 \mu \mathrm{m} .2 \mathrm{r} / w$ is the normalized disk radius.

cross the barrier and the two films act as one single fused film. In the opposite case the screening currents in the two films are independent on each other and no cross talk is allowed. In the intermediate situations, a fraction of the screening currents in one film crosses the barrier and circulates in the other film and vice versa. It is clear that, for a given transverse field and a given junction and electrodes geometry, the transition from the fused to the independent films regime can be controlled via the barrier transparency, i.e., in our case by the Josephson current density $J_{c}$. The determination of the magnetic field distribution in a system made by two superconducting film forming a planar JTJ is a very complex task, since it also involves the Josephson equations. The analysis becomes even more difficult when the JTJ is biased (the distribution of the bias current peaks near the film edges) and the junction dimensions exceed the Josephson penetration depth $\lambda_{J}=\sqrt{\hbar / 2 e \mu_{0} d_{e} J_{c}}$.

However, following Ref. 14, we can argue that in the independent films regime, the intensity of the in-plane magnetic field felt by a planar JTJ placed at the borders of two superconducting films can exceed by several times the value of the applied transverse field. In fact, the screening currents flowing within a depth $\lambda_{L, b}$ in the top surface of the bottom film and those flowing within a depth $\lambda_{L, t}$ in the bottom surface of the top film have opposite directions. Consequently, the associated magnetic fields add in the barrier plane and a more efficient modulation of the JTJ critical current is achieved.

\section{EXPERIMENTS}

In order to prove the advantages to use a transverse field rather than an in-plane one, we have measured the transverse magnetic diffraction patterns $I_{c}\left(H_{\perp}\right)$ of planar high quality JTJs having different geometries, sizes, and critical current densities. The samples were placed on the axis of a superconducting coil surrounded by a $\mathrm{Pb}$ shield and a cryoperm can in order to attenuate the earth magnetic field. The magnetic field produced by the coil was calculated through COMSOL MultiPhysics numerical simulations. 


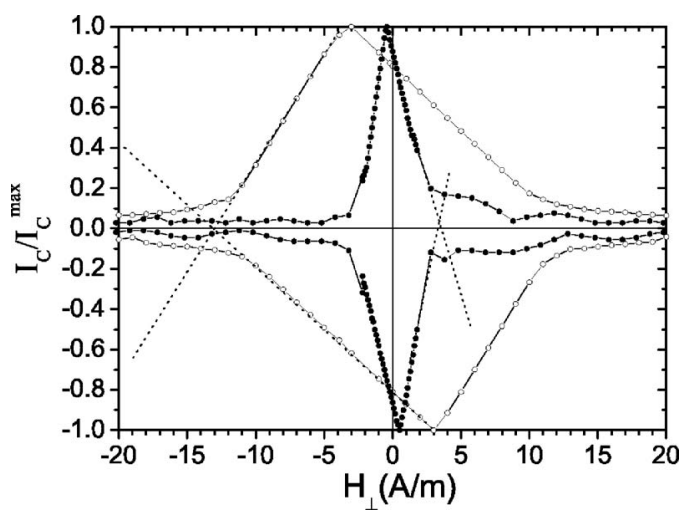

FIG. 2. Magnetic diffraction patterns measured in a transverse field $H_{\perp}$ of two overlap-type junctions $L \times W=500 \times 4 \mu \mathrm{m}^{2}$. The closed circles refer to a $\mathrm{Nb} / \mathrm{Al}_{\text {ox }} / \mathrm{Nb}$ JTJ having $J_{c}=60 \mathrm{~A} / \mathrm{cm}^{2}, d_{e}=180 \mathrm{~nm}$, and $\lambda_{J} \sim 50 \mu \mathrm{m}$. The open circles refer to a $\mathrm{Nb} / \mathrm{AlN} / \mathrm{NbN}$ with $J_{c}=400 \mathrm{~A} / \mathrm{cm}^{2}, d_{e}$ $=240 \mathrm{~nm}$, and $\lambda_{J} \sim 20 \mu \mathrm{m}$. The dotted lines show the procedure used to determine the junction critical field $H_{c}^{\perp}$.

\section{A. Overlap-type junctions}

Figure 2 compares the diffraction patterns measured in a transverse field of two overlap-type junctions having the same geometry and dimensions, but different critical current density $J_{c}$. The junctions have been fabricated with the trilayer technique in which the junction is realized in the window opened in $\mathrm{SiO}_{2}$ insulating layer. The thicknesses of the base, top, and wiring layer are 200, 100, and $400 \mathrm{~nm}$, respectively. Details of the fabrication process can be found in Ref. 16. The junction length is $L=500 \mu \mathrm{m}$, while the width is equal to $4 \mu \mathrm{m}$. The base and top electrode widths are 540 and $506 \mu \mathrm{m}$, respectively. The so called "idle region," i.e., the overlapping of the wiring layer onto the base electrode is about $3 \mu \mathrm{m}$ all around the barrier. In Fig. 2 the junction critical currents have been normalized to their maximum values in order to make the comparison easier. The closed circles refer to a $\mathrm{Nb} / \mathrm{Al}_{\mathrm{ox}} / \mathrm{Nb}-\mathrm{Nb}$ tunnel junction having a critical current density $J_{c}=60 \mathrm{~A} / \mathrm{cm}^{2}$, while the open circles refer to a $\mathrm{Nb} / \mathrm{AlN} / \mathrm{NbN}-\mathrm{Nb}$ sample with $J_{c}$ $=400 \mathrm{~A} / \mathrm{cm}^{2}$. As expected, considering that the field lines associated to the screening current are perpendicular to the electrodes edges, the shape of these $I_{c}$ versus $H_{\perp}$ curves looks very like that of the expected for long one-dimensional overlap-type junctions when a uniform external field $H_{\|}$is applied in the barrier plane in the direction perpendicular to the junction length. In fact, according to the analysis of Refs. 17 and 18, for small field values (Meissner regime) the critical current $I_{c}$ decreases linearly with the applied field $I_{c}\left(H_{\|}\right)=I_{c}(0)\left(1-\left|H_{\|}\right| / H_{c}^{\|}\right)$, where $H_{c}^{\|}$is the critical field at which $I_{c}$ would vanish if flux quanta did not start to enter the junction barrier. The skewness seen in the experimental $I_{c}-H_{\perp}$ curves (being larger for the sample having the larger $J_{c}$ ) is due to the self-field produced by the bias current $I$ flowing in a close-by superconducting strip in the chip circuitry. The skewness does not prevent us from measuring the junction critical fields $H_{c}^{\perp}$ determined by linearly extrapolating the branches starting at maximum critical current to $I$ $=0$ (dotted lines in Fig. 1); in fact, when $I=0$, the bias current self-field effects vanish. The critical field $H_{c}^{\|}$for a long
TABLE I. Relevant parameters for two overlap-type Josephson tunnel junctions whose transverse magnetic diffraction patterns are shown in Fig. 2.

\begin{tabular}{lccccc}
\hline \hline \multicolumn{1}{c}{ Sample } & $\begin{array}{c}J_{c} \\
\left(\mathrm{~A} / \mathrm{cm}^{2}\right)\end{array}$ & $\begin{array}{c}d_{e} \\
(\mathrm{~nm})\end{array}$ & $L / \lambda_{J}$ & $\begin{array}{c}H_{c}^{\perp} \\
(\mathrm{A} / \mathrm{m})\end{array}$ & $\begin{array}{c}H_{c}^{\|}=H_{c}^{F}+H_{c}^{\mathrm{FP}} \\
(\mathrm{A} / \mathrm{m})\end{array}$ \\
\hline $\mathrm{Nb} / \mathrm{Al}_{\mathrm{ox}} / \mathrm{Nb}$ & 60 & 180 & $\sim 10$ & 3.2 & $36+58=94$ \\
$\mathrm{Nb} / \mathrm{AlN} / \mathrm{NbN}$ & 410 & 240 & $\sim 25$ & 13 & $36+131=167$ \\
\hline \hline
\end{tabular}

one-dimensional junction in presence of an in-plane external field applied perpendicular to the long junction dimension $L$ can be expressed as the sum of two terms

$$
\begin{aligned}
H_{c} & =H_{c}^{F}+H_{c}^{\mathrm{FP}}=\frac{\Phi_{0}}{\mu_{0} d_{e} L}+2 \lambda_{J} J_{c} \\
& =\frac{\Phi_{0}}{\mu_{0} d_{e}}\left(\frac{1}{L}+\frac{1}{\pi \lambda_{J}}\right)=\frac{\Phi_{0}}{\mu_{0} d_{e} L}+\sqrt{\frac{2 \Phi_{0} J_{c}}{\pi \mu_{0} d_{e}}} .
\end{aligned}
$$

The first term $H_{c}^{F}$ in Eq. (2) dominates in low $J_{c}$ samples for which $L \ll \pi \lambda_{j}$ and corresponds to the critical field of a pointlike junction which exhibits the well known Fraunhofer diffraction pattern. The second term $H_{c}^{\mathrm{FP}} \propto \sqrt{J_{c} / d_{e}}$ becomes dominant in the high $J_{c}$ regime when $L \gg \pi \lambda_{j}$. It was first introduced by Ferrell and Prange ${ }^{19}$ in order to describe the self-field limiting effects in long inline-type junctions.

Table I reports the values of $J_{c}$ and $d_{e}$ used to predict the critical field $H_{c}^{\|}$from to Eq. (2). The last two columns allow the comparison between $H_{c}^{\perp}$ and $H_{c}^{\|}$. We observe that for both samples $H_{c}^{\|}>H_{c}^{\perp}$ and the ratio $H_{c}^{\|} / H_{c}^{\perp}$ changes from about 30 to about 13 when we move from the low to the high $J_{c}$ junction. The data in Table I unambiguously indicate that a transverse field can be more effective than an in-plane one and, remembering that the two samples have the same geometrical details, the effect of a transverse field weakens as the junction transparency increases.

\section{B. Annular junctions}

Further, we have measured the static properties of several $\mathrm{Nb} / \mathrm{Al}_{\mathrm{OX}} / \mathrm{Nb}-\mathrm{Nb}$ annular JTJs having the same critical current density $J_{c}=60 \mathrm{~A} / \mathrm{cm}^{2}\left(\lambda_{J}=50 \mu \mathrm{m}\right)$, the same width $\Delta R=4 \mu \mathrm{m}$, but different radius $R$ ranging from 80 to $500 \mu \mathrm{m}$. The fabrication details are the same as those for the overlap-type JTJs discussed previously. The diffraction patterns in transverse magnetic field will be reported elsewhere $^{20}$; here we focus our interest on the values of the critical fields.

The analogous of Eq. (2) for a one-dimensional annular junction having radius $R$ in an in-plane field is ${ }^{21}$

$$
H_{c}=H_{c}^{B}+H_{c}^{\mathrm{MM}}=2.404 \frac{\Phi_{0}}{2 \pi \mu_{0} d_{e} R}+R J_{c} .
$$

Again we have a contribution $H_{c}^{B}$, independent on the Josephson current density $J_{c}$, typical of small and intermediate radius annular JTJs immersed in a uniform in-plane magnetic field which results in a periodic radial field $H_{r}(\theta)$ $\propto \cos \theta$ felt by the junction. ${ }^{22,23}$ In such case, the $I_{c}$ versus $H_{\|}$ curve follows a Bessel, rather than a Fraunhofer, behavior (2.404 is the argument corresponding to the first minimum of the zero-order Bessel function). The second term $H_{c}^{\mathrm{MM}}=R J_{c}$ 
TABLE II. Relevant parameters of the $\mathrm{Nb} / \mathrm{Al}_{\mathrm{ox}} / \mathrm{Nb}$ annular Josephson tunnel junctions used in the experiments.

\begin{tabular}{cccc}
\hline \hline $\begin{array}{c}R \\
(\mathrm{~mm})\end{array}$ & $R / \lambda_{J}$ & $\begin{array}{c}H_{c}^{\perp} \\
(\mathrm{A} / \mathrm{m})\end{array}$ & $\begin{array}{c}H_{c}^{\|}=H_{c}^{B}+H_{c}^{\mathrm{MM}} \\
(\mathrm{A} / \mathrm{m})\end{array}$ \\
\hline 0.08 & $\sim 1.6$ & 2.7 & $18+48=66$ \\
0.25 & $\sim 5$ & 1.5 & $6+150=156$ \\
0.32 & $\sim 6.4$ & 1.3 & $4+192=196$ \\
0.50 & $\sim 10$ & 1.2 & $3+300=303$ \\
\hline \hline
\end{tabular}

in Eq. (3) was numerically found by Martucciello and Monaco; ${ }^{21}$ considering that $H_{c}^{\mathrm{MM}} / H_{c}^{B}=\left(R / \lambda_{J}\right)^{2} / 2.404$, it becomes dominant when $R \gg R_{m}=\sqrt{2.404} \lambda_{J} .{ }^{9,23}$ For the given $d_{e}$ and $J_{c}$, Eq. (3) has a minimum when $R=R_{m}$ and linearly increase with $R$ when $R \gg R_{m}$. The last two columns of the Table II report, respectively, the transverse critical field $H_{c}^{\perp}$ measured for four annular junctions having different radii and the expected parallel critical field $H_{c}^{\|}$according to Eq. (3) with $J_{c}=60 \mathrm{~A} / \mathrm{cm}^{2}$ and $d_{e}=180 \mathrm{~nm}$.

For all samples we observe once again that $H_{c}^{\|}>H_{c}^{\perp}$ and that the ratio $H_{c}^{\|} / H_{c}^{\perp}$ changes from about 25 to about 250 when we increase the ring diameter, i.e., the top and bottom film widths, confirming that the effect of a transverse field strengthens as the electrode widths increases. In particular, for the three largest rings, having the so called Lyngby geometry, ${ }^{24}$ i.e., the base and top electrode widths match the ring diameter, this ratio is proportional to $R$. [The smallest ring $\left(R / \lambda_{J} \approx 1.6\right)$ has the base electrode width equal to $540 \mu \mathrm{m}$ that is considerably larger than the ring diameter $160 \mu \mathrm{m}$.

\section{CONCLUDING REMARKS}

We have measured the transverse magnetic diffraction pattern of planar JTJs having different geometries, sizes, and barrier transparencies. Our measurements clearly indicate that a magnetic field is more effective to modulate the junction critical current $I_{c}$ when applied perpendicularly (rather than parallel) to the junction plane provided the JTJ is fabri- cated close to the borders of superconducting films with a large aspect ratio. This is due to screening (or Meissner) currents induced by the transverse field that circulate mainly on the film surface borders which in turn behave as intrinsic control lines. We suggest that a transverse magnetic field can be usefully exploited in those applications where the Josephson critical current and the Fiske resonances need to be suppressed.

${ }^{1}$ A. Barone and G. Paternò, Physics and Applications of the Josephson Effect (Wiley, New York, 1982).

${ }^{2}$ P. W. Anderson and J. M. Rowell, Phys. Rev. Lett. 10, 2130 (1963).

${ }^{3}$ B. D. Josephson, Adv. Phys. 14, 419 (1965).

${ }^{4}$ M. Weihnacht, Phys. Status Solidi 32, K169 (1969).

${ }^{5}$ J. M. Rowell, Phys. Rev. Lett. 11, 200 (1963).

${ }^{6}$ V. P. Koshelets, S. V. Shitov, L. V. Filippenko, A. M. Baryshev, H. Golstein, T. de Graauw, W. Luinge, H. Schaeffer, and H. van de Stadt, Appl. Phys. Lett. 68, 1273 (1996).

${ }^{7}$ E. P. Houwman, J. G. Gijsbertsen, J. Flokstra, and H. Rogalla, Physica C 183, 339 (1991)

${ }^{8}$ T. Nagatsuma, K. Enpuku, F. Irie, and K. Yoshida, J. Appl. Phys. 54, 3302 (1983).

${ }^{9}$ N. Martucciello, J. Mygind, V. P. Koshelets, A. V. Shchukin, L. V. Filippenko, and R. Monaco, Phys. Rev. B 57, 5444 (1998).

${ }^{10}$ I. Rosenstein and J. T. Chen, Phys. Rev. Lett. 35, 303 (1975).

${ }^{11}$ A. F. Hebard and T. A. Fulton, Phys. Rev. Lett. 35, 1310 (1975).

${ }^{12}$ E. H. Brandt and J. R. Clem, Phys. Rev. B 69, 184509 (2004).

${ }^{13}$ A. C. Rose-Innes and E. H. Rhoderick, Introduction to Superconductivity (Pergamon, Oxford, 1969), Par. 8.7.

${ }^{14}$ S. L. Miller, K. R. Biagi, J. R. Clem, and D. K. Finnemore, Phys. Rev. 31, 2684 (1985)

${ }^{15}$ M. Kobayashi and Y. Sugiyama, IEEE Trans. Magn. 26, 297 (1990).

${ }^{16}$ P. N. Dmitriev, I. L. Lapitskaya, L. V. Filippenko, A. B. Ermakov, S. V. Shitov, G. V. Prokopenko, S. A. Kovtonyuk, and V. P. Koshelets, IEEE Trans. Appl. Supercond. 13, 107 (2003).

${ }^{17}$ C. S. Owen and D. J. Scalapino, Phys. Rev. 164, 538 (1967).

${ }^{18}$ A. Barone, W. J. Johnson, and R. Vaglio, J. Appl. Phys. 46, 3628 (1975).

${ }^{19}$ R. A. Ferrell and R. E. Prange, Phys. Rev. Lett. 10, 479 (1963).

${ }^{20}$ R. Monaco, J. Mygind, M. Aaroe, and V. P. Koshelets, "Annular Josephson tunnel junctions in a transverse magnetic field," (unpublished).

${ }^{21}$ N. Martucciello and R. Monaco, Phys. Rev. B 54, 9050 (1996).

${ }^{22}$ N. Grønbech-Jensen, P. S. Lomdahl, and M. R. Samuelsen, Phys. Lett. A 154, 14 (1991)

${ }^{23}$ N. Martucciello and R. Monaco, Phys. Rev. B 53, 3471 (1996).

${ }^{24}$ A. Davidson, B. Dueholm, B. Kryger, and N. F. Pedersen, Phys. Rev. Lett. 55, 2059 (1985). 